\title{
Grietas en las bibliotecas azules. La relación entre Lasso de la Vega y Miguel Artigas durante la Guerra Civil Española (1936-1939)
}

\section{Cracks in the blue libraries. The relationship between Lasso de la Vega and Miguel Artigas during the Spanish Civil War (1936-1939)}

\author{
Luís Blanco Domingo \\ lmblanco@unizar.es \\ Departamento de Ciencias de la Documentación e Historia de la Ciencia de la \\ Universidad de Zaragoza
}

\section{Resumen}

El presente artículo analiza un episodio poco conocido de la política bibliotecaria del bando sublevado durante la Guerra Civil, que muestra las desavenencias y recelos suscitados entre Miguel Artigas y Javier Lasso de la Vega, dos de sus más significados representantes, en tres vertientes: los servicios de lecturas dirigidas a los soldados, la pugna por alcanzar puestos de responsabilidad en la administración del Nuevo Estado franquista, y la posibilidad de implantar el modelo de encabezamientos anglosajón.

\section{Palabras clave}

Miguel Artigas; Javier Lasso de la Vega; Política bibliotecaria; Guerra Civil Española

\section{Abstract}

This article analyzes a little-known episode of the library policy of the rebellious side during the Civil War, which shows the disagreements and misgivings raised between Miguel Artigas and Javier Lasso de la Vega, two of its most significant representatives, in three aspects: the services of readings directed to the soldiers, the struggle to reach positions of responsibility in the administration of the "Nuevo Estado", and the possibility of implanting the model of Anglo-Saxon headings. 


\section{Keywords}

Miguel Artigas; Javier Lasso de la Vega; Library policy; Spanish Civil War

Recibido: 15/04/2018

Aceptado: 15/06/2018

DOI: $\underline{\text { http://dx.doi.org/10.5557/IIMEI9-N16-027048 }}$

Descripción propuesta: Blanco Domingo, Luis, 2018. Grietas en las bibliotecas azules. La relación entre Lasso de la Vega y Miguel Artigas durante la Guerra Civil Española (1936-1939). Métodos de Información, 9(16), 27-48

\section{Introducción}

La Biblioteca de la Universidad de Zaragoza durante la guerra civil se convirtió en el escenario de un conflicto más profesional que personal entre dos de los más significados representantes de la biblioteconomía española de la época: Miguel Artigas y Javier Lasso de la Vega. Sobre el primero existe una reciente biografía muy centrada en su actividad anterior a la guerra (Hoz Regules 2017), mientras que Lasso ha sido estudiado con profusión por Martínez Montalvo (Martínez Montalvo 1999, 2000). Con respecto a las vicisitudes del Centro académico, son muy válidas las aportaciones de Remedios Moralejo, si bien están integradas dentro de una historia global de la institución. (Moralejo Álvarez 1985, 1996).

Si bien Artigas se encontraba de forma accidental en el territorio aragonés debido al disfrute de sus vacaciones estivales, y mostró desde el inició su intención de colaborar con el bando sublevado, Lasso regresaría más tarde a España, puesto que el golpe de estado le sorprendió en Portugal, lo que provocó algunas acusaciones de tibieza y falta de convicción en su apoyo a los sublevado. Curiosamente, ambos se sucedieron en el control y gestión de las competencias del Cuerpo Facultativo de Archivos, Bibliotecas y Museos en dos etapas diferenciadas, cuyos lapsos cronológicos los marca el mes de marzo de 1938. 
El momento de inflexión que supone esa fecha explica el tránsito desde una administración pensada para ganar la guerra, estructurada en Juntas Técnicas, a otra que progresivamente se encargará de gestionar la victoria, organizada en Ministerios. En uno de ellos, el de Educación, dirigido por Pedro Sáinz Rodríguez, Lasso de la Vega será designado como Jefe de Archivos y Bibliotecas. Ese nombramiento será quizá el detonante principal del distanciamiento que experimentarán a partir de ese momento, y que culminará con el proceso de depuración a que se verá sometido tras el fin de la contienda bélica.

Las siguientes páginas procuran recorrer los espacios y formas de ese desencuentro.

\section{Los inicios. E1 Servicio de Lecturas para el Soldado}

Tras jurar fidelidad al "Movimiento Nacional" en la Universidad de Zaragoza en presencia de su Rector, Gonzalo Calamita, una de las primeras iniciativas de Artigas fue la implantación del Servicio de Lecturas para el Soldado. Este ente tuvo sus orígenes durante el desarrollo de la I Guerra Mundial, orientado a la consecución de tres objetivos fundamentales: facilitar un medio de distracción al soldado herido; influenciar y exaltar su ánimo combatiente por medio de lecturas dirigidas y debidamente filtradas, y por último convertirse en el vehículo imprescindible de una actividad terapéutica novedosa, la biblioterapia, como complemento para la cicatrización no tanto de sus heridas físicas como mentales.

Durante la guerra civil, ambos bandos dispusieron de organismos que dinamizaron distintas actuaciones dirigidas a mejorar la situación anímica de los soldados tanto en los frentes de combate como en los hospitales que recibían a los heridos. Sin embargo, la eficacia y profundidad de los entes republicanos, uno de ellos gestionado por el Servicio de Cultura Popular desde Madrid $^{1}$ y el Servei de Bibliotheques del Front, creado por la Generalitat de

\footnotetext{
${ }^{1}$ En su constitución destacó sobremanera Teresa Andrés, funcionaria del Cuerpo Facultativo de Archiveros, Bibliotecarios y Arqueólogos tras ganar la oposición el 8 de agosto de 1931. Destinada primeramente al Museo Arqueológico de León, fue adscrita el 9 de septiembre del mismo año como jefa del Archivo del Palacio Real. Durante la guerra ocupó cargos de relevancia en el Ministerio de Instrucción Pública, desarrollando una intensa labor de extensión bibliotecaria y fomento de la lectura en frentes y hospitales, siendo la autora de un pequeño manual técnico,
} 
Catalunya ${ }^{2}$ superaban con creces la iniciativa del bando nacional amparada bajo el nombre de Servicio de Lecturas del Soldado ${ }^{3}$, no sólo por la menor implicación de los centros de poder y el escaso apoyo político recibido, sino también por la concepción de la lectura como una actividad perniciosa que debía ser sometida a rigurosas dosis de control y depuración antes de aceptarla, y el rechazo frontal a su virtualidad como canal educativo y de promoción social.

La institución gestionada por el bando sublevado experimentó dos épocas muy distintas (Blanco Domingo 2017). Hasta el nombramiento de Lasso de la Vega como jefe de Archivos, Bibliotecas y Museos en marzo de 1938, Artigas dirigió un proyecto caracterizado por la provisionalidad, la improvisación, el escaso o nulo apoyo oficial y la carencia de infraestructuras adecuadas. En esta fase, Lasso de la Vega era un subordinado más, encargado de la gestión del Servicio en el Distrito Universitario de Sevilla, desde el que manifiesta en repetidas ocasiones un malestar latente por cuanto considera que, ante la teórica e inminente toma de Madrid, debía recuperar su antiguo puesto de bibliotecario en la Universidad Central.

Lasso, el máximo experto español en biblioterapia y uno de los pioneros en su introducción en el país, quedaba relegado a un puesto secundario e incómodo. Sin embargo, a partir de marzo, con la asunción de la responsabilidad y el control de la política bibliotecaria, dota al Servicio de un reglamento estructurado con voluntad de permanencia, en un contexto bélico más estable y beneficioso para el bando sublevado, en el que el centro de influencia y gestión bibliotecaria había gravitado de Zaragoza a Vitoria, mientras que Artigas se había trasladado a Santander con el propósito de ampliar los apoyos de los hispanistas e intelectuales foráneos a la causa nacional.

Indicaciones para la organización de bibliotecas de frentes, hospitales y cuarteles, Valencia: Cultura Popular, 1937, destinado a reglamentar la constitución y el funcionamiento de las mismas. Para ampliar su biografía, véase Gomez Andres, Antonio. Teresa Andrés. Biografía. Valencia: Universitat de Valencia, 2013.

2 Cugueró, M., Boada, M.T. y Allué, V. El Servei de Bibliotheques del Front: 1936-1939. Barcelona: Diputació de Barcelona, 1995. ALLUÉ, Vicenç. "El Servei de Bibliotheques del Front, epopeia cultural del segle XX". Item: Revista de biblioteconomia i documentació, No. 44 (Setembre-desembre), 2006, págs. 67-112

${ }^{3}$ García Ejarque, L. Historia de la lectura pública en España. Gijón: Trea, 2000, pp. 187 y ss. 


\section{Un nombramiento cuestionado}

La rivalidad soterrada y mantenida en el tiempo entre Miguel Artigas y Javier Lasso de la Vega tuvo su momento álgido cuando Sáinz Rodríguez decidió elegir como Jefe del Servicio de Archivos, Bibliotecas y Registro de la Propiedad Intelectual al segundo tras la organización ministerial de 1938 (Martínez Montalvo 2000: 109-119) Lasso, ayudante del nuevo ministro de Educación Nacional en la Cátedra de Bibliología de la Universidad de Madrid, mantenía una relación más o menos cordial con Artigas. Ambos representaban dos concepciones distintas de la Biblioteconomía, sobre todo, desde el punto de vista de la influencia social. Mientras que Artigas respondía a los parámetros del bibliotecario clásico, más preocupado de los procesos técnicos y de organización de fondos que de la atención a los usuarios, Lasso, sobre todo gracias a su formación en bibliotecas americanas, tenía un concepto más "orteguiano" de la función de los profesionales, en el que la interacción y la función social e higienista de las bibliotecas prevalecía sobre cualquier otra condición, sin desdeñar su conocimiento de las nuevas ideas sobre la organización bibliotecaria y la lectura pública. Precisamente por esta idea más "moderna", actualizada y "social" de la Biblioteca justificaba Sáinz Rodríguez su elección, a pesar de que ello le produjo cierto distanciamiento con el bibliotecario turolense.

"He de decir que el nombramiento de Javier Lasso me produjo un cierto rozamiento con un amigo querido, Miguel Artigas, bibliotecario de la Biblioteca Menéndez Pelayo, pero en realidad él se hizo cargo de lo que motivó mi decisión. Tú perteneces a los bibliotecarios clásicos -le expliqué-, y tu función, cuando se pueda, ha de ser la dirección de la Biblioteca Nacional de Madrid, pero para esta organización y en esta época, creo más útil la colaboración de Javier Lasso. Él se convenció y quedamos tan amigos". (Sáinz Rodríguez 1978: 260).

Algunos autores, entre los que sobresale Escolar Sobrino, argumentan que la preferencia se debía a ese aporte de ideas renovadoras, tan necesarias para modificar sustancialmente tanto los programas escolares, básicos en el proceso de adoctrinamiento pedagógico en el que se encontraba inmerso el Nuevo Estado, como en los servicios bibliotecarios (Escolar Sobrino 1989: 293). 
Artigas y Sáinz Rodríguez se habían conocido en Santander años atrás, cuando éste veraneaba en la localidad cántabra de Viveda. Gracias a su buena relación con Adolfo Bonilla, único testamentario de Menéndez Pelayo, Sáinz Rodríguez empezó a frecuentar la Biblioteca Menéndez Pelayo en el momento en que Artigas fue designado director de la misma. Ambos se dedicaron a organizar y clasificar la numerosa documentación legada por el célebre polígrafo, y entre ellos surgió una buena amistad.

La frustración que le supuso a Artigas verse relegado a un segundo plano en el organigrama ministerial, sobre todo después de que él asumiera desde Zaragoza la labor de diseñar la política bibliotecaria del bando rebelde en un momento de enorme dificultad y desorden, se acrecentaría paulatinamente debido al impulso legislador de Lasso y a la proliferación de normas que afectaban a servicios y actividades implementadas por Artigas, como por ejemplo, y de forma muy significativa, el Servicio de Lectura del Soldado, organismo en el que había sido designado por el propio Artigas para que asumiera su dirección en el distrito controlado por la Universidad de Sevilla (Alted Vigil 1984: 53-56).

Las medidas legislativas en materia de bibliotecas que siguieron al nombramiento de Lasso fueron muy numerosas. Entre ellas destacan el nacimiento de los Patronatos Provinciales de Bibliotecas, la introducción de la ficha única con encabezamiento variable, el desarrollo de secciones de libre acceso en las bibliotecas, la dinamización y gestión del préstamo de libros, la adopción de medidas que establecieran los cauces adecuados para una colaboración estrecha y fructífera entre las bibliotecas y las escuelas, o la aparición de la Clasificación Decimal Universal (Martínez Montalvo 1999: 183-296).

Sin embargo, pese a la enjundia y profundidad de todas estas decisiones normativas, dirigidas a sentar las bases de una reforma de la gestión bibliotecaria que transcendiera la propia eventualidad del contexto bélico, la mayoría no tuvieron traslación práctica ni continuidad debido a las propias necesidades bélicas, la dispersión geográfica de los facultativos que deberían asumir su desarrollo, ocupados además en funciones ajenas a su labor profesional, y un indisimulado escepticismo sobre la oportunidad temporal de su implementación, que se unía a algunos recelos existentes entre algunos 
facultativos, no sólo en Artigas, por el nombramiento de Lasso de la Vega como Jefe de la Sección de Archivos, Bibliotecas y Museos (Escolar Sobrino 1987: 222)

A ello debemos añadir el concepto oficial de cultura que emanaba del bando nacional, oscilante entre la apuesta por difundir los ideales nacionalcatólicos y el recelo con el que se contemplaba a los libros, entendidos como factores de discordia y responsables indirectos de la intervención militar, a los que era necesario someter a un control exhaustivo. ${ }^{4}$

Lasso concebía las bibliotecas como centros de formación y adiestramiento de los individuos para evitar que se intoxicaran mediante la difusión de ideas subversivas, al mismo tiempo que se convertían en lugares de conformación ideológica del patriota a través de modelos de conducta respaldados por lecturas dirigidas. La utilidad social de las bibliotecas era por tanto considerable. ${ }^{5}$

Tampoco es desdeñable el considerable impacto de las veleidades republicanas de Lasso, y su cercanía a círculos masónicos sevillanos, circunstancias bien conocidas por todos los facultativos del Cuerpo. Ello sin embargo no le impidió ser elegido para el cargo, ante el escepticismo, cuando no la sorpresa de muchos de sus compañeros. Sus intervenciones en actividades profesionales durante la II República fueron constantes, integrado en organizaciones como la Asociación de Bibliotecarios y Bibliógrafos de España o el II Congreso Internacional de Bibliotecas y Bibliografía, mientras que Artigas mantenía un silencio expectante o una neutralidad activa y cercana a grupos conservadores. Un artículo publicado por Lasso de la Vega en 1934 por la revista Boletín de Bibliotecas y Bibliología, que él mismo había creado, con el expresivo título de Política bibliotecaria, manifestaba que los bibliotecarios

\footnotetext{
${ }^{4}$ Hipólito Escolar señala como una de las causas fundamentales de la ausencia de nuevas bibliotecas en la zona nacionalista "el miedo a los efectos destructores de muchos libros" entre las autoridades militares y religiosas. Escolar Sobrino, Hipólito (1989: 296)

${ }^{5}$ La comparativa con los objetivos perseguidos por la política bibliotecaria republicana ha originado una interesante polémica historiográfica entre aquellos que, como Hipólito Escolar, consideran que fue un mero instrumento político tendente a favorecer autores y corrientes ideológicas afines, frente a otros que, como Ana Martínez Rus, rechazan la presencia de sectarismo, y hablan de un concepto de biblioteca abierta y plural. Escolar Sobrino, Hipólito (1987: 32-33). Martínez Rus, Ana (2001: $115-118$ y 2014)
} 
españoles consideraban que sin libros, sin prensa y sin bibliotecas públicas España no podría ser un país democrático. Pero además insistía en la capacidad educativa y emancipadora de la lectura cuando advertía que "no se puede olvidar que los regímenes autoritarios pueden vivir y desenvolverse con que sólo los dirigentes sean cultos y estén dotados de la necesaria preparación. En los regímenes democráticos, allí donde la elección de los gobernantes y la dirección de la política están encomendadas al ciudadano, es de todo punto necesario educar, capacitar a la masa, a la muchedumbre, al pueblo" (Lasso de la Vega 1934: 14)

Resulta extraordinariamente revelador de ese progresivo distanciamiento, que paulatinamente se iba transformando en cierta acritud, el testimonio de Pedro Muguruza, Comisario General del Servicio de Defensa del Patrimonio Artístico $\mathrm{Nacional}^{6}$ en una carta dirigida desde Zaragoza al entonces ministro Pedro Sáinz Rodríguez durante su inspección a las zonas monumentales afectadas por la guerra. Tras manifestar su sorpresa por la petición de Artigas de solicitar su ingreso en el Servicio de Patrimonio creado por el gobierno de Burgos, como afirma Tomás García Diego, encargado del Servicio de Bellas Artes en un informe elevado a la superioridad de la Junta Técnica, (SDPAN. Correspondencia. 5 de octubre de 1937). Muguruza constata tanto la ambición y necesidad de reubicación de Artigas como la frialdad y escasa sintonía con Lasso de la Vega (SDPAN. Correspondencia, 11 de julio de 1938)

A pesar de su interés, y de una primera decisión que aprobaba su incorporación al Servicio Artístico de Vanguardia, una oportuna reorganización administrativa declaró nula su adscripción en septiembre de 1937 (AGA. Educación. Vitoria 7 de septiembre de 1937. Caja 31/4753)

Sobre este escenario impactaba además la obsesión pedagógica que presidía buena parte de las iniciativas legislativas desarrolladas por Lasso, buscando tanto adaptar el rol de las bibliotecas a las necesidades de información demandadas por los potenciales usuarios, como integrarlas en el nuevo diseño de la formación académica del alumnado.

\footnotetext{
${ }^{6}$ Creado por una circular de 2 de julio de 1938, este Servicio dividía el territorio ocupado por el bando nacional en siete circunscripciones, integrando Zaragoza la llamada zona de Levante, dirigida por José María Muguruza. Alted Vigil, Alicia (1984: 82)
} 
No obstante, el contexto en el que se aprueban las medidas, la carencia de soporte financiero para su crecimiento y los propios recelos que tanto la figura de Lasso como la marcada influencia anglosajona de sus medidas despertaban tanto entre compañeros del Cuerpo como sobre todo entre las autoridades, provocaron que la mayor parte de ellas no traspasaran la barrera de lo puramente teórico y bienintencionado. ${ }^{7}$

Pese a esa frialdad constatable con la que se recibió su nombramiento, Lasso intentó consensuar una respuesta unánime del Cuerpo Facultativo al desdén con que las autoridades militares trataban la mayor parte de sus actuaciones. Por ello, buscó la complicidad de Artigas y Gómez del Campillo con la redacción, bajo criterios comunes, de una propuesta programática que pretendía mostrar la importancia de su presencia en la nueva administración. Se basaba en una petición del aumento del personal auxiliar, la creación de un órgano consultivo y jefaturas provinciales que superaran el "autocratismo desmoralizador" de la etapa republicana, la redacción de modelos de partes de trabajo unificadores y la búsqueda de un edificio arquetípico y funcional para albergar los distintos establecimientos. Sin embargo, las expectativas pronto se vieron frustradas puesto que las prioridades bélicas y las tesis oficialistas marginaban conscientemente tanto a archivos como a bibliotecas, frente al mayor impacto propagandístico de las obras de arte, a las que prestaban mayor atención y entusiasmo.

Las discrepancias entre Artigas y Lasso tenían un componente fundamentalmente profesional, y no derivaban únicamente en la rivalidad por obtener cargos relevantes en el nuevo organigrama que se estaba gestando. Representaban dos concepciones distintas de la gestión y organización bibliotecaria, más clásica en el caso de Artigas y con mayor presencia de las nuevas corrientes de la biblioteconomía, de inspiración anglosajona, en Lasso. Comenzaba a perfilarse una visión de las bibliotecas alejada de la iconografía

\footnotetext{
7 Escolar considera que la labor legislativa de Lasso "adolece de ingenuidad, en parte por el conocimiento parcial que tenía de la realidad bibliotecaria española, y en parte por la precipitación con que quiso difundir o realizar teorías leídas en libros extranjeros", si bien señala que "su labor en Vitoria fue muy importante, y desde luego muy superior a la que desarrollaron los bibliotecarios de la zona republicana" Escolar Sobrino, Hipólito (1987: 222-223)
} 
tradicional, en la que aspectos como su tratamiento como mero depósito de libros o la percepción del bibliotecario dedicado casi en exclusiva al proceso técnico y distanciado de sus usuarios se cuestionaban con creciente éxito. ${ }^{8}$

\section{La polémica sobre las normas de descripción}

Precisamente relacionado con esas diferentes visiones de la realidad bibliotecaria española, uno de los elementos de fricción entre Lasso y Artigas tuvo como motivo la redacción de normas para confeccionar catálogos para las bibliotecas y la pretensión de implantarlas en todos los centros y establecimientos dependientes del Cuerpo. Los objetivos que perseguía Lasso con su publicación eran varios. En primer lugar, cumplir con el compromiso de publicar unas reglas destinadas a confeccionar los catálogos de las bibliotecas administradas por el Cuerpo Facultativo, que había sido adquirido en la introducción a las Instrucciones para la redacción de los Catálogos de las Bibliotecas Públicas del Estado, aprobadas por la Real Orden de 31 de julio de 1902. En segundo término, atender las prerrogativas emanadas del Decreto de 22 de abril de 1938, que permitía el acceso libre de los usuarios a los catálogos en cédulas sueltas, alfabéticos de autores, científicos, de títulos y los ordenados por sistema de diccionario, pero también consideraba la posibilidad de ofrecer a los facultativos una serie de reglas que permitieran su formación y redacción.

Este revolucionario Decreto fue sin duda el espaldarazo legal que Lasso necesitaba para publicar, con la colaboración de Cesáreo Goicoechea, las instrucciones destinadas a generalizar la formación y redacción de catálogos en las bibliotecas españolas (Lasso de la Vega, J. y C. Goicoechea 1938)

Pero sobre todo, en su ánimo pesaba la necesidad de dotar a todos los facultativos de un instrumento de descripción metódico y multidisciplinar,

\footnotetext{
8 Algunos autores, como Martínez Montalvo interpretan esta nueva visión como una fase fundamental que explica el tránsito desde la biblioteconomía tradicional a las modernas concepciones del análisis documental. No obstante, no debemos obviar el influjo de los postulados ideológicos franquistas, marcados por las referencias higienistas, mediadoras y sectarias del rol del bibliotecario, y el modelo de dirigismo cultural que predicaban, factores que limitan considerablemente tanto el alcance de las reformas como su carácter modernizador. Martínez Montalvo, Esperanza (1999).
} 
capaz de adaptarse a cualquier disciplina científica, que revitalice su función social y responda a las nuevas demandas planteadas por los usuarios.

"La profesión de bibliotecario, por otra parte, encuentra su técnica más específica y respetable cuando se implanta un sistema de clasificación científica ... Los bibliotecarios de Casinos, Círculos etc no profesionales, no pueden abordar esta labor porque ahí comienza la faceta más visible al público de nuestra profesión y ahí se inicia el respeto hacia ella". (Ibidem, p. 7.)

Para ello Lasso, con la estrecha colaboración de Cesáreo Goicoechea, trató de establecer una serie de objetivos concretos, claramente definidos en el prólogo de sus Reglas. En primer lugar, proporcionar un catálogo unificado de autores, título y materias en un solo índice que por un lado simplifique y facilite su uso por parte del público, y al mismo tiempo se convierta en un instrumento eficaz a los bibliotecarios para redactar el Catálogo-Diccionario. En segundo lugar, los autores constatan que se trata tan sólo de unas reglas genéricas e introductorias, aunque claras y precisas, que buscan un fin eminentemente práctico: la agilización del trabajo bibliotecario. Pese a ello, sí consideran que las Reglas aportan una guía para la utilización de los subencabezamientos, que permite solventar la enorme disparidad existente entre los distintos modelos practicados en las bibliotecas hispanoamericanas a la hora de concebir sus catálogos.

En tercer lugar, una clara vocación internacional no sólo por sus reiteradas apelaciones a su extensión a toda Hispanoamérica, sino a las fuentes y publicaciones en las que se basa. Nos encontramos ante una adaptación de la tabla de encabezamientos creada por Sears en Estados Unidos, ampliamente utilizada sobre todo en el mundo anglosajón. El carácter interdisciplinar, la universalidad y la flexibilidad que la definen posibilitan su adaptación a cualquier centro bibliotecario, sin que ello signifique que sea capaz de resolver todos los problemas que se susciten. Del mismo modo, sus apéndices se inspiran en las Instrucciones de la Biblioteca Vaticana, de notoria influencia estadounidense, y en la monografía Rules for filing cards de la Biblioteca Carnegie de Pittsburgh. ${ }^{9}$

\footnotetext{
${ }^{9}$ Ibidem, pp. 10-11. Se trata de la obra Rules for filing cards in the dictionary catalogues of the Carnegie library of Pittsburgh, elaborada por el propio centro, y cuya primera edición apareció en 1915 compilada por
} 
Lasso de la Vega le envía una carta a Artigas demandándole su opinión respecto a la metodología que han empleado para confeccionar las Instrucciones a contemplar en la realización de los catálogos diccionarios, que ha realizado conjuntamente con Cesáreo Goicoechea. (ABHMV. Fondo Lasso de la Vega, 10 de octubre de 1938). Ante la carencia de una respuesta, el 20 de octubre reitera su interés en conocer sus consideraciones al respecto, sin conocer que el día 15 Artigas había manifestado acuse de recibo en el que proclamaba su absoluta y perentoria necesidad, sobre todo ahora que ya no existían lo que calificaba de "estorbos burocráticos" que impedían su implantación. (ABMV. Fondo Lasso de la Vega, 15 de octubre de 1938)

La tarea de normalización de los catálogos acometida por Lasso de la Vega crecía sin descanso. En noviembre compartía con Artigas haber finalizado la traducción de la clasificación decimal de Dewey, y la intención de llevar conjuntamente a la imprenta ambas instrucciones, a las que añadiría el respaldo legislativo necesario que permitiera su aplicación bien de forma conjunta o separada. (ABHMV. Fondo Lasso de la Vega, 3 de noviembre de 1938).

Sin embargo, Artigas no parece muy entusiasmo por la posibilidad de adaptar las instrucciones al panorama bibliotecario español, sobre todo sin haber acometido algunos trabajos previos que permitan su introducción, y anuncia la necesidad de mantener una extensa conversación al respecto con Lasso. (ABHMV. Fondo Lasso de la Vega, 14 de enero de 1939).

Debemos tener además en cuenta la irrupción de ciertos recelos intelectuales por cuanto Artigas puede considerarse el pionero en España de la idea de confeccionar un catálogo colectivo, inspirado en el que realizara la Biblioteca Real de Berlín, y que él mismo pudo analizar durante su estancia en Alemania. Su comunicación a la Asamblea de Archiveros, Bibliotecarios y Arqueólogos de 1923 proponía que se emprendiese ese trabajo siguiendo las coordenadas marcadas por el modelo prusiano. Pero no sólo limitó su estrategia a la mera teorización. Con su nombramiento para dirigir la Biblioteca Nacional,

Margaret Mann. Sus contenidos se basaban en la cuarta edición de la obra de Charles Cutter Rules for a dictionary catalogue 
emprendió la reproducción mecánica del catálogo alfabético de autores, con el objetivo de ofrecer al público un instrumento útil y al mismo tiempo enviar las fichas resultantes a las principales bibliotecas españolas para que, tras su confrontación con sus fondos, remitiesen las de los libros que no poseía la Nacional y formar el que calificaba como Catálogo general del tesoro bibliográfico español.

Coexisten por tanto dos visiones que giran no sólo sobre el calendario de aplicación de las Instrucciones, sino también sobre los principios dogmáticos que las sustentan. Mientras que Artigas se muestra reacio a una implementación inmediata sin haber acometido con anterioridad una serie de actuaciones en el marco bibliotecario que allanen y faciliten su desarrollo, sobre todo en lo relacionado con las papeletas de autores, Lasso entiende éstas como innecesarias, y un freno a sus innovadoras propuestas.

Por ello, conmina a Artigas, aprovechando que las Instrucciones se estaban imprimiendo, a que le responda a una serie de preguntas concretas, relacionadas con determinados aspectos. En primer lugar si cree que debe aplicarse el sistema de Diccionario a todas las bibliotecas o por el contrario, su escepticismo sobre los resultados del proceso le conduce a considerar que no debe introducirse en ningún caso o bien sólo en aquellos establecimientos que dispusieran de unas papeletas referidas a los autores. En segundo lugar, si estima que ni la metodología seguida ni el modelo teórico utilizado, de hondas raíces estadounidenses, resultan adecuados. Y si fuera así, que identifique cuáles son los errores básicos y qué corriente biblioteconómica o autor concreto debería a su juicio tomarse como referente. Y por último, todas aquellas puntualizaciones y sugerencias que estime oportunas y justifiquen las reservas que manifiesta Artigas sobre la eficacia de la propuesta. (ABHMV. Fondo Lasso de la Vega, 18 de enero de 1939).

Las diferencias, más allá de una aparente relación cordial, no tardaron en aparecer. La política de hechos consumados practicada por Lasso había provocado el aumento de la desconfianza en Artigas, con lo que el tono epistolar se tornó más agrio y menos complaciente. Lasso manifiesta su contrariedad con el tono displicente y el desdén con que Artigas responde a su misiva, y lo argumenta con varias razones. En primer lugar, porque Artigas había abogado siempre, "tanto en público y en privado" por el sistema de 
clasificación de diccionario, recordando especialmente una reunión entre Menéndez Pidal, José Castillejo y ambos en el Centro de Estudios Históricos en la que, sin renunciar a las posibilidades del Catálogo Diccionario, Lasso defendía como sistema de clasificación más correcto el decimal. Incluso tras una conversación mantenida en Sevilla, en la que "cediendo a sus gustos", Lasso le anunció la intencionalidad de confeccionar el mencionado Catálogo, asumiendo la recomendación acertada de redactar en primer lugar unas tablas que permitieran seguir las instrucciones.

En segundo lugar, Lasso entiende que han utilizado las fuentes bibliográficas y los criterios de autoridad fundamentales en la materia tratada, teniendo como intención última "proporcionar al Cuerpo un instrumento de trabajo útil", razón por la cual se han decidido a dar curso a su impresión. Si Artigas interpretara que no fueran aplicables abandonarían el proyecto, por lo que espera le indique con más detalle el alcance de sus apreciaciones y/o modificaciones. Si se tratara tan sólo de problemas de índole doctrinal, se declara dispuesto a suspender inmediatamente el trabajo para acomodarlo al autor o a las teorías que le indicara, habida cuenta de "su superior autoridad en la materia". (ABHMV. Fondo Lasso de la Vega, 18 de enero de 1939).

La respuesta de Artigas es harto elocuente. Comienza reiterando su idea de que el sistema de Diccionario es preferible básicamente para el público. Si se dispusiera de la clasificación decimal para los profesionales de la biblioteca o para "estudiosos ya cultos" sería mucho mejor, le revestiría de un carácter más científico. No participa sin embargo del "desdén con que trata el alfabetismo", y sus reservas, que el mismo considera inexistentes, apuntan precisamente a la necesidad de disponer de un buen índice alfabético. Las causas, fundadas en su experiencia y conocimiento de las bibliotecas españolas y su situación, se centran en la inexistencia de catálogos alfabéticos que no sólo simplificarían y agilizarían la clasificación posterior por materias, sino que además servirían de catálogo topográfico, tan esencial desde su punto de vista en las bibliotecas. Entiende que los catálogos para los encabezamientos necesitan una revisión siguiendo la metodología utilizada en la Biblioteca Nacional bajo su coordinación, y que les ocupó durante tres años.

No obstante, estima que las instrucciones son una herramienta útil. Pero considera que no se pueden aplicar de forma generalizada hasta que las 
bibliotecas no estén catalogadas y ordenadas de forma conveniente, y sobre todo, y en relación con esta primera premisa, cuando tras realizar de forma efectiva y fehaciente el recuento de todos los funcionarios del Cuerpo activos, se nombren comisiones volantes que finalicen con el inventariado de todos los fondos de las bibliotecas. Los miembros de dichas comisiones podrían incluso asesorar a los bibliotecarios que lo precisen de las dificultades que encierra el desarrollo del sistema de Diccionario.

Otro de los problemas que denuncia se refiere a las infraestructuras y los edificios, a la necesidad de realizar obras en numerosas bibliotecas, puesto que la escasez de espacio y las malas condiciones de muchos de los centros impiden el control adecuado de los libros que componen su fondo, al obligar en numerosas ocasiones a ubicarlos en lugares que dificultan su colocación y acceso.

No parece muy conforme con la premura en publicar la obra, puesto que consideraba que hubiera resultado mucho más útil y adecuado imprimir unas cuantas copias para que sirvieran de ensayo en unas cuantas bibliotecas seleccionadas, entre las cuales la Biblioteca Universitaria de Sevilla podría erigirse en el arquetipo de centro, puesto que ya estaban perfectamente definidos y unificados los encabezamientos. De todas formas, y siguiendo lo acontecido en la Biblioteca Nacional, hubieran surgido una serie de problemas que conducirían a la adopción de una fórmula mixta, esto es, la alfabetización de las materias en el catálogo y la signatura, con dos cifras decimales previas en las papeletas, para determinar un poco más las materias y ayudar a la clasificación por Títulos.

Consciente de esos problemas, Artigas había logrado la concesión de dos pensiones para que dos bibliotecarios acudieran a Italia, concretamente a la Biblioteca Vaticana ${ }^{10}$, con el objetivo de estudiar sus sistemas de instrucciones y tablas de clasificación, muy útiles sobre todo teniendo en cuenta la escasa

\footnotetext{
10 Norme per il catalogo degli stampati, Ciudad del Vaticano, 1931. La Biblioteca Vaticana realizó una compilación de las reglas de catalogación para libros impresos que sintetiza las corrientes europeas con las novedosas aportaciones de la biblioteconomía americana, están estructuradas en forma de catálogo diccionario basado en las reglas de Cutter. Garrido Arilla, María Rosa (2000: 219)
} 
operatividad y vigencia de unos catálogos que no se habían modernizado ni en cuanto a su lenguaje ni en cuanto a la elección de materias.

A pesar de las objeciones de Artigas, la obra tuvo un éxito considerable y una rápida y amplia difusión, debido probablemente a ser la primera lista de encabezamientos de materias diseñada para la formación de catálogosdiccionario publicada en España. Sin embargo, la propia biblioteca de la Universidad de Zaragoza atestigua la enorme dificultad que suponía su aplicación en el contexto bélico actual. La Memoria de 1938 expone que, a pesar de su reconocida utilidad para facilitar la información bibliográfica, existen dificultades "de carácter económico y personal", que impiden implantar el novedoso sistema. (AGA. Educación. Memoria ...,1938). El modelo que se utilizaba hasta ese momento era el denominado capsintético, ${ }^{11}$ que agrupaba las papeletas bibliográficas en unos ficheros que podrían contener índices de títulos o de materias. En la mencionada memoria, Sánchez Viejo, director de la biblioteca, señala sus preferencias por el segundo, argumentando una mayor flexibilidad y eficacia, sobre todo si al mismo tiempo se complementa con el recurso a claves de búsqueda como las referencias geográficas (AGA. Educación. Memoria ..., 1938).

\section{Un epitafio doloroso: el proceso de depuración de Lasso de la Vega}

Sin embargo, el relevo de Sáinz Rodríguez por Ibáñez Martín modificó sustancialmente el mapa de sus relaciones. Su caída en desgracia llevó aparejada la de Lasso de la Vega y el ascenso de Artigas a la Dirección General de Archivos y Bibliotecas el 25 de agosto de 1939, ya finalizada la guerra. Ello produjo no sólo su reincorporación a la dirección de la Biblioteca de la Universidad Central y a su labor docente en las enseñanzas de Biblioteconomía en la Facultad de Filosofía y Letras, sino la reapertura del proceso de depuración que, iniciado en octubre de 1936, quedó en suspenso

\footnotetext{
11 Se trata de un sistema ideado en 1851 por Pierre Pinçon, bibliotecario y conservador de la Biblioteca de Santa Genoveva de París, en el que la información bibliográfica aparece en forma de fichas perforadas y agrupadas en una caja con el fin de evitar el recurso constante a los catálogos y registros y garantizar su conservación.
} 
temporal en noviembre de 1937 al no haberse encontrado pruebas suficientes de vinculación con la masonería. ${ }^{12}$

El mismo día que tenía previsto comenzar la vista, el 3 de noviembre de 1939, una orden del Ministerio de Educación Nacional declaraba la exención de ser sometido al proceso por haber desempeñado un cargo de confianza en el Ministerio de Educación. Por este motivo, el proceso estuvo congelado hasta el 26 de marzo de 1942. Sin embargo, el tribunal calificador, formado por Miguel Artigas, Miguel Gómez del Campillo y Blas Taracena, que habían manifestado siempre sus suspicacias por el ascenso y la notoriedad alcanzadas por Lasso, no cejó en el empeño de proseguir con el encausamiento. Su probado acercamiento a la masonería fue la causa esgrimida por Artigas para elevar una propuesta de sanción al Tribunal para la Represión de la Masonería y del Comunismo, que fue atendida con dos consecuencias: la suspensión temporal del implicado en todos los cargos dependientes del Ministerio de Educación, y que se dictara el pliego de cargos.

Pese a que el 12 de junio la resolución es favorable a Lasso, no sólo no se le restituye en su puesto de director de la Biblioteca Universitaria de Madrid, sino que también se le deja fuera de la plantilla y es destinado al Servicio de las Bibliotecas Populares de Madrid según orden ministerial de 14 de octubre de 1942, lo que podemos interpretar como un síntoma de degradación de cargos y una suerte de "destierro intelectual" instigado tanto por Artigas como por Miguel Gómez del Campillo.

Lasso, convencido de que se había cometido una enorme injusticia, remitió varias cartas tanto al Rector de la Universidad de Madrid como al ministro Ibáñez Martín solicitando aclaraciones y que intercediesen por él, lo que tendría éxito transcurridos tres años. El 10 de agosto de 1945 recupera su cargo como director de la Biblioteca de la Universidad de Madrid, y unos

\footnotetext{
${ }^{12}$ Escolar señala al respecto que Lasso "después de la guerra sufrió por su amistad con Sáinz Rodríguez más que persecución, vigilancia, que el apartó de las fuentes de poder, por lo que su actividad se orientó, por un lado, hacia los trabajos internacionales en la FIO y, por otro, a la publicación de numerosos artículos y obras sobre bibliotecas e información y a la colaboración con empresas editoriales, como Editorial Labor". Escolar Sobrino, Hipólito. (1989: 297)
} 
meses más tarde, en noviembre, su caso es definitivamente sobreseído, aunque la sospecha de masón le perseguiría buena parte de su vida.

\section{Conclusiones}

La censura provocada por la Guerra Civil Española impactó también sobre el Cuerpo Facultativo de Archiveros, Bibliotecarios y Arqueólogos. Dos de las figuras más destacadas y con mayor peso intelectual en el colectivo, Miguel Artigas y Javier Lasso de la Vega, mostraron su apoyo al bando sublevado, más temprano en el caso del primero que del segundo.

Su actividad durante el conflicto discurrió de forma paralela, vinculada a organismos bibliotecarios surgidos ad hoc, urgentes y de carácter finalista, condicionados por las necesidades bélicas. Podemos hablar de varias etapas diferentes y contradictorias, relacionadas con el grado de influencia de las distintas sensibilidades que integraban el bando nacional. Desde el golpe de estado de julio de 1936 hasta marzo de 1938, Artigas dirigirá la política bibliotecaria desde la Universidad de Zaragoza integrada en unas estructuras administrativas endebles e inestables, pensadas para ganar la guerra, mientras que a partir de marzo de 1938, se asentarán los principios y las instituciones que van a conformar el Nuevo Estado franquista, orientados a gestionar la ya próxima victoria militar. Franco nombra su primer gobierno, y entre las carteras ministeriales incluye la de Educación Nacional, encomendada a Pedro Sáinz Rodríguez, intelectual católico de hondas concepciones monárquicas.

Entre los distintos órganos auxiliares que crea se encuentra la Jefatura del Servicio de Archivos, Bibliotecas y Registro de la Propiedad Intelectual. Tras algunas dudas, designa a Lasso de la Vega, con quien había compartido docencia en la Universidad de Madrid, frente a la candidatura de Artigas, e inconscientemente nos muestra la existencia de diferencias conceptuales y teóricas entre ambos. El primero, de sólida formación anglosajona, muestra una idea más "social” y con tintes "modernos" frente a Artigas, más apegado a la tradición biblioteconómica europea clásica. Precisamente esa idea de modernidad es la que, según refiere el propio Sáinz Rodríguez, decanta la balanza a favor del sevillano. 
Esta elección puede ser interpretada como el detonante de sus desencuentros. Artigas, relegado a un segundo plano en el nuevo organigrama ministerial, se centrará en dinamizar el apoyo de los intelectuales foráneos e hispanistas al bando sublevado desde Santander. No obstante, la presunta rivalidad entre ambos debe ser interpretada desde el ámbito puramente profesional, lo que no impide que esté trufada de algunos desaires de carácter personal. Y además, las desavenencias ya procedían de situaciones anteriores.

Hasta marzo de 1938, Lasso fue un subordinado de Artigas en el Servicio de Lecturas para el Soldado en el distrito universitario de Sevilla, sin que ello le impidiera mostrar su descontento por una situación que consideraba injusta y poco acorde con sus capacidades. A partir de esa fecha, será Lasso quien reglamente el órgano, y quien asuma la jefatura de todos los funcionarios del Cuerpo, incluido el propio Artigas.

A esta circunstancia debemos añadir una interesante controversia teórica sobre la unificación de los encabezamientos de materia para su aplicación en las bibliotecas. La publicación de las Normas junto con Cesáreo Goicoechea, origina un intercambio epistolar entre Lasso y Artigas sobre la oportunidad de introducirlas en los centros españoles. Artigas no se muestra muy partidario de su generalización, por cuanto implantar este modelo requeriría un sistema bibliotecario capaz de asumirlo, inventarios reales de los fondos que contienen, y conocer realmente los funcionarios de los que dispone el Cuerpo Facultativo, circunstancias harto complicadas en tiempos de guerra.

Pese a que en algunos momentos parecieron establecer un frente común con el objetivo de reivindicar el papel del Cuerpo Facultativo, siempre secundario y relegado por la mayor trascendencia propagandística del patrimonio artístico frente al documental y el concepto del libro como elemento dañino que se debe erradicar, el relevo de Sáinz Rodríguez y el ascenso del falangista Ibáñez Martín al Ministerio una vez finalizada la guerra agranda sus diferencias. En 1939, Artigas asume la Dirección General de Archivos y Bibliotecas, y es uno de los que conforman el tribunal que reabre el proceso de depuración de Lasso de la Vega. El expediente se dilata en el tiempo. Pese a que la normativa consideraba al encausado exento por haber desempeñado un cargo de confianza, Artigas solicitó una propuesta de sanción por las veleidades 
masónicas y republicanas de Lasso, que le condenaría a la indiferencia y al destierro intelectual hasta el año 1945.

Un corolario sin duda significativo de unas discrepancias soterradas pero constantes entre dos de los más significados representantes de la biblioteconomía española, cuyas trayectorias intelectuales se vieron alteradas por la Guerra Civil. La necesidad de transformar las bibliotecas en centros de combate, complementarios y auxiliares de los avances militares, subordinaba el resto de sus actividades. Pero sin embargo, podemos vislumbrar, sobre todo a partir de marzo de 1938, los cimientos ideológicos y teóricos sobre los que se iba a sustentar la política bibliotecaria del Nuevo Estado.

\section{Fuentes primarias}

Archivo de la Biblioteca Histórica Marqués de Valdecilla (ABHMV). Fondo Lasso de la Vega. Carta de Lasso de la Vega a Miguel Artigas pidiendo su opinión sobre el Catálogo-Diccionario redactado junto con Goicoechea. Vitoria 10 de octubre de 1938.

ABHMV. Fondo Lasso de la Vega. Carta de Miguel Artigas a Lasso de la Vega sobre la necesidad de reformas. Santander 15 de octubre de 1938.

ABHMV. Fondo Lasso de la Vega. Carta de Lasso de la Vega a Miguel Artigas sobre la posibilidad de llevar a la imprenta la traducción de la CDU de Dewey. Vitoria 3 de noviembre de 1938.

ABHMV. Fondo Lasso de la Vega Carta de Miguel Artigas a Lasso de la Vega sobre las Instrucciones y el Catálogo-Diccionario redactados por éste. Santander 14 de enero de 1939.

ABHMV. Fondo Lasso de la Vega. Carta de Lasso de la Vega a Miguel Artigas con el fin de concretar sus objeciones a su Catálogo-Diccionario. Vitoria 18 de enero de 1939.

ABHMV. Fondo Lasso de la Vega. Carta de Lasso de la Vega a Miguel Artigas con el fin de concretar sus objeciones a su Catálogo-Diccionario. Vitoria 18 de enero de 1939.

Archivo General de la Administración (AGA). Educación. Oficio de la Comisión de Cultura de la Junta Técnica de estado que declara nula la adscripción de Miguel Artigas al Servicio Artístico de Vanguardia. Vitoria 7 de septiembre de 1937. Caja 31/4753 
AGA. Educación. Memoria de la Biblioteca de la Universidad de Zaragoza 1938. Caja $31 / 5459$

Servicio de Defensa del Patrimonio Nacional (SDPAN). Correspondencia. Tomás García Diego. Informe del Consejero de Cultura encargado del Servicio de Bellas Artes, Burgos, 5 de octubre de 1937

SDPAN. Correspondencia. Carta de Pedro Muguruza a Pedro Sáinz Rodríguez: Zaragoza, 11 de julio de 1938.

\section{Bibliografía}

ALLUÉ, V., 2006. El Servei de Bibliotheques del Front, epopeia cultural del segle XX. Item: Revista de biblioteconomia i documentació, nº .44 , págs. 67-112

ALTED VIGIL, A., 1984. Política del Nuevo Estado sobre el patrimonio cultural y la educación durante la Guerra Civil Española. Madrid: Dirección General de Bellas Artes y Archivos, Centro Nacional de Información Artística, Arqueológica y Etnológica

BLANCO DOMINGO, L., 2017. Libros como trincheras. El Servicio de Lecturas del Soldado de la Biblioteca de la Universidad de Zaragoza durante la Guerra Civil (1936-1939), Revista General de Información y Documentación, 27 (2), 433-470. ISSN 1988-2858.

CUGUERÓ, M., BOADA, M.T. y ALLUÉ, V. 1995. El Servei de Bibliotheques del Front: 1936-1939. Barcelona: Diputació de Barcelona.

ESCOLAR SOBRINO, H., 1987. La cultura durante la Guerra Civil. Madrid: Alhambra.

ESCOLAR SOBRINO, H., 1989. El compromiso intelectual de bibliotecarios y editores. Madrid: Fundación Germán Sánchez Ruipérez.

GARRIDO ARILLA, Ma R., 2000. Contienda por el control documentario: etapas pretécnica y técnica en catalogación. Cuadernos De Documentación Multimedia, vol. 10, pp. 211-223.

GOMEZ ANDRES, Antonio. Teresa Andrés. Biografía. Valencia: Universitat de Valencia, 2013 
HOZ REGULES, J. de la, 2017. Miguel Artigas. De la Biblioteca Menéndez Pelayo a la dirección de la Biblioteca Nacional. Madrid: FUE.

LASSO DE LA VEGA, J., 1934. Política bibliotecaria. Boletín de Bibliotecas y Bibliografía, vol. I, 2, pp. 9-16.

LASSO DE LA VEGA, J. y C. GOICOECHEA. 1938. Reglas para la formación y redacción de los catálogos-diccionarios en las bibliotecas, seguidas de una lista de encabezamientos de materias y numerosos apéndices. Santander: Aldus.

MARTÍNEZ MONTALVO, E., 1999. Aportaciones a la teoría e bistoria de la documentación en España: vida y obra de Javier Lasso de la Vega (1892-1930). Tesis Doctoral dirigida por López Yepes, José. Departamento de Biblioteconomía y Documentación. Universidad Complutense de Madrid.

MARTÍNEZ MONTALVO, E., 2000. Investigación y producción cientifica en documentación: la obra de Javier Lasso de la Vega (1892-1990). Madrid: Fragua.

MARTÍNEZ RUS, A. 2001. La politica del libro durante la II República: socialización de la lectura. [En línea]. Tesis doctoral dirigida por Martínez Martín, Jesús A. Madrid: Universidad Complutense de Madrid, Facultad de Geografía e Historia. [Consulta: 07/04/2018]. Disponible en http://biblioteca.ucm.es/tesis/ghi/ucm-t25567.pdf

MARTÍNEZ RUS, A. 2014. La persecución del libro: hogueras, infierno y buenas lecturas (19361951). Madrid: Trea

MORALEJO ÁLVAREZ, R., 1985. La Biblioteca Universitaria de Zaragoza". Aragón Cultural, vol. 10. pp. 20-22.

MORALEJO ÁLVAREZ, R., 1996. La Biblioteca Universitaria de Zaragoza. [En línea] Boletín de la $A N A B A D$, vol. 46, no. 3, pp. 319-350. Disponible en http://dialnet.unirioja.es/descarga/articulo/51030.pdf. [Consulta: 16 de mayo de 2018].

SÁINZ RODRÍGUEZ, P., 1978. Testimonio y recuerdos. Barcelona: Planeta 